\section{Brustkrebsmortalität und fettreduzierte Ernährung}

In einer Studie der WHI (Women's Health Initiative) hatte eine fettreduzierte Ernährung bei postmenopausalen Frauen das Auftreten von Brustkrebs nach 8,5 Jahren vermindert. Ziel dieser Analyse war es, den Langzeiteinfluss einer solchen Diät auf tumorbedingte und Gesamtmortalität über 16 Jahre zu prüfen.

Z wischen 1993 und 1998 wurden an 40 US-Zentren 48.835 postmenopausale Frauen mit unauffälliger Mammografie, ohne vorausgegangenes Mammakarzinom und u.a. einem Fettanteil von über $32 \%$ an der Gesamtkalorienzufuhr aufgenommen. $40 \%$ der Frauen $(n=19.541)$ erhielten randomisiert eine intensive $\mathrm{Er}$ nährungsberatung und -anleitung mit dem Ziel, die Fettaufnahme auf $20 \%$ der

Energiezufuhr $\mathrm{zu}$ begrenzen und stattdessen mehr Obst, Gemüse und

Körner zu essen. Die Vergleichsgruppe $\quad(60 \%$; $\mathrm{n}=29.294)$ erhielt nur eine allgemeine Beratung. Die interventio-

Gesunde Ernährung senkte die Mortalität. nelle Studienphase dauerte median 8,5 Jahre, die Gesamtstudiendauer liegt jetzt bei median 16,1 Jahren. In der Interventionsgruppe nahmen Fettzufuhr und Körpergewicht signifikant ab (beides $\mathrm{p}<$ 0,001). Die Einhaltung der Diät wurde durch ein intensives Beratungsprogramm sichergestellt und durch nachfolgende Fragebogen-Erhebungen überwacht.

In der 8,5 Jahre dauernden Interventionsphase wurden insgesamt 1.764 Mammakarzinome registriert. Wie schon früher berichtet, war der Anteil unter der Ernährungsumstellung um $8 \%$ niedriger. Die jetzige Analyse konzentrierte sich auf tumorspezifische und Gesamtmortalität der Patientinnen mit Mammakarzinom.

Bei Frauen mit fettreduzierter Ernährung traten in der Interventionsphase zwar weniger brustkrebsbedingte Todesfälle auf als im Vergleichsarm, der
Unterschied war jedoch nicht signifikant $(0,016$ vs. $0,024 \%$ pro Jahr; $p=0,08)$. Wohl aber war die Gesamtmortalität der Brustkrebspatientinnen unter der fettreduzierten Diät signifikant niedriger $(0,025$ vs. $0,038 \%$ pro Jahr; $\mathrm{p}=0,02)$.

Während der Gesamtdauer von 16,1 Jahren (Interventions- plus Follow-upPhase) traten in beiden Armen insgesamt 3.030 Mammakarzinome auf. Die Zahl der brustkrebsbedingten Todesfälle wurde hier leicht gesenkt (0,035 vs. 0,039\% pro Jahr; $p=0,41)$. Ein deutlicherer Nutzen zeigte sich aber wieder bei der Gesamtmortalität ( 0,085 vs. $0,11 \%$ pro Jahr; $\mathrm{p}=0,01)$. Subgruppenanalysen ergaben, dass Frauen mit erhöhtem Fettanteil bei der Kalorienzufuhr oder solche mit höherem Bauchumfang stärker von der Ernährungsumstellung profitierten.

Fazit: Die fettreduzierte Ernährung war in einer großen, randomisierten, kontrollierten WHI-Studie bei postmenopausalen Frauen mit weniger Todesfällen nach dem Auftreten von Brustkrebs assoziiert.

Brigitte Schalhorn

Chlebowski RT et al. Low-fat dietary pattern and breast cancer mortality in the Women's Health Initiative randomized controlled trial. J Clin Oncol. 2017;35(25):2919-26.

\title{
Mammakarzinom-Subtypen für Entscheidung zur Radiatio relevant
}

In einer Analyse der Phase-III-Studie EORTC 10994/BIG 1-00 wurden klinische und pathologische Faktoren für ein lokoregionäres Rezidiv (LRR) bei Brustkrebspatientinnen mit neoadjuvanter Chemotherapie untersucht, weil diese bei der Entscheidung über eine lokoregionale Bestrahlung helfen könnten.

In der Studie EORTC 10994/BIG 1-00 waren Frauen mit großen operablen oder lokal fortgeschrittenen Mammakarzinomen nach neoadjuvanter Chemotherapie (NAC) und Operation teilweise bestrahlt worden. Patientinnen mit Hormonrezeptor-positivem Tumor nahmen zudem adjuvant 5 Jahre Tamoxifen ein, bei HER2-positiven Tumoren konnten sie adjuvant Trastuzumab erhalten. Nach median 4,4 Jahren hatten von 1.553 auswertbaren Patientinnen 67 ein LRR entwickelt (kumulative Inzidenz 4,9\%) - gegenüber früheren Ergebnissen wenige, evtl. wegen der Fortschritte bei der Radiotherapie, der Therapie mit Tamoxifen und Trastuzumab. Trotz niedriger Inzidenz ließen sich multivariat 2 wesentliche Prädiktoren für ein LRR identifizieren: der Brustkrebs-Subtyp und das pathologische Ansprechen auf die NAC (jeweils $\mathrm{p}<0,0001)$. Bei tripel-negativem Brustkrebs war das LRR-Risiko gegenüber Luminal-A-Tumoren deutlich erhöht (Hazard Ratio [HR] 6,44; 95\%-Konfidenzintervall $[95 \%-\mathrm{KI}] 2,83-14,69)$, ähnlich bei HER2-positivem Karzinom ohne Trastuzumab-Gabe (HR 6,26; 95\%-KI 2,81-
13,93). Etwas günstiger, aber immer noch gegenüber Luminal-A-Tumoren deutlich erhöht, fiel das LRR-Risiko bei HER2-positiven Karzinomen mit TrastuzumabTherapie aus (HR 3,37, 95\%-KI 1,1010,34 ). Fanden sich $\geq 4$ positive Lymphknoten, war das LRR-Risiko mehr als verdoppelt (HR 2,43; 95\%-KI 1,34-4,40).

Fazit: Die Ergebnisse legen nahe, neben einem positiven nodalen Befund nach neoadjuvanter Chemotherapie auch den Mammakarzinom-Subtyp in die Entscheidung über eine adjuvante Strahlentherapie einzubeziehen. Friederike Klein

Gillon P et al. Factors predictive of locoregional recurrence following neoadjuvant chemotherapy in patients with large operable or locally advanced breast cancer: An analysis of the EORTC 10994/ BIG 1-00 study. Eur J Cancer. 2017;79:226-34. 